\title{
SHELLFRAT - UM SISTEMA COMPUTACIONAL PARA ANÁLISE DE TRINCAS NÃO PASSANTES EM ESTRUTURAS DE SUPERFÍCIE
}

\author{
LUIZ ANTONIO DE SOUZA ${ }^{1}$ \\ NELSON F. F. EBECKEN ${ }^{2}$
}

SOUZA, L. A. de; EBECKEN, N. F. F. SHELLFRAT - Um sistema computacional para análise de trincas não passantes em estruturas de superfície. Semina. Ci. Exatas/Tecnol. Londrina, v. 21, n. 4, p. 9-16, dez. 2000.

RESUMO: Este trabalho apresenta o sistema computacional SHELLFRAT, baseado no método dos elementos finitos (MEF) para análise de trincas não-passantes em estruturas de superfície. A estrutura é discretizada por elementos de casca e a trinca é simulada por elementos de mola, que permitem representar trincas com forma, direção e profundidades variáveis. O sistema pode processar análises: em regime elástico ou elásto-plástico, considerações de modos mistos de fratura, simular a propagação da trincas por fadiga e prever a vida útil da estrutura. Como resultado da análise são avaliados os fatores de intensidade de tensão (FIT), o integral $\mathrm{J}$, o deslocamento de abertura de trinca (COD-Crack Opening Displacement), geometria final da trinca e número de ciclos de carregamento.

PALAVRAS-CHAVE: elementos finitos, cascas, trincas, modos de fratura, fadiga.

1

\section{INTRODUÇÃO}

Estruturas de cascas são com usadas geralmente em engenharia, para a construção de reservatórios, tanques de combustíveis, tubulações e vasos de pressão, estruturas off-shore, usinas nucleares, cascos de navios, laminas de turbinas, equipamentos espaciais e muitas outras aplicações. Estes tipos de estruturas estão geralmente submetidas a severas condições de trabalho, que além de outras causas levam ao desenvolvimento de trincas ao longo de sua espessura. Isto leva a uma perda parcial da capacidade resistente da estrutura, sem no entanto, levá-la a um colapso instantâneo, pois a parte remanescente da seção trincada pode ainda absolver e transmitir esforços. Entretanto, uma análise rigorosa e precisa deve ser efetuada com a finalidade de avaliar as reals condições da estrutura, assim como determinar parâmetros que indiquem a velocidade de propagação e a geometria crítica da trinca. Estes parâmetros sao geralmente o fator de intensidade de tensão propostos por Irwin (1957), o integral-J (Rice, 1968) e o COD.

A avaliação destes parâmetros em trincas nãopassantes é um problema tipicamente tridimensional e levando-se em conta as mais diversas combinações de geometria e carregamento, tornase impossível a utilização de métodos analíticos. Como alternativa utiliza-se de métodos numéricos como, por exemplo, o método dos elementos finitos e o método dos elementos de contorno. Entretanto, a abordagem do problema utilizando-se um procedimento tridimensional envolvem um grande volume de dados, discretizações complexas e enorme esforço computacional, principalmente quando se deseja uma análise em regime elastoplástico ou a previsão da vida útil da estrutura.

Uma alternativa para este tipo de problema e a utilização do modelo de linha de molas (MLM), que foi proposto por Rice \& Levy (1972). Posteriormente foi associado ao elemento finito de casca por German, Kumar e Lorenzi (1982), tornando-se uma ferramenta versátil, segura e de fácil utilização.

\section{O MODELO DE LINHA DE MOLAS}

O modelo de linha de molas (Figura 1) foi concebido por Rice \& Levy (1972) e devido a sua versatilidade e qualidade de seus resultados, vários

\footnotetext{
1 Universidade Estadual de Londrina, CTU/DE - Cx. Postal 6001. CEP 86051-990, Londrina, PR.

${ }^{2}$ COPPE/UfRJ, Prog, de Eng. Civil, Cx. Postal 68506, CEP 21945-870, Rio de Janeiro, RJ.
} 
pesquisadores o têm utilizado e contribuído para o seu desenvoivimento (Rice, 1973; Delale \& Erdogan,
1981; Desvaux, 1985; Souza \& Ebecken, 1987; Souza, 1993; Olowokere, 1997; Skallured, 1999).

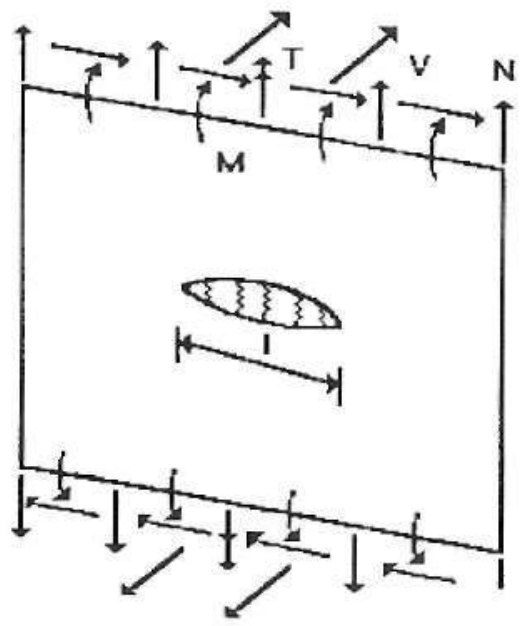

[1.a]

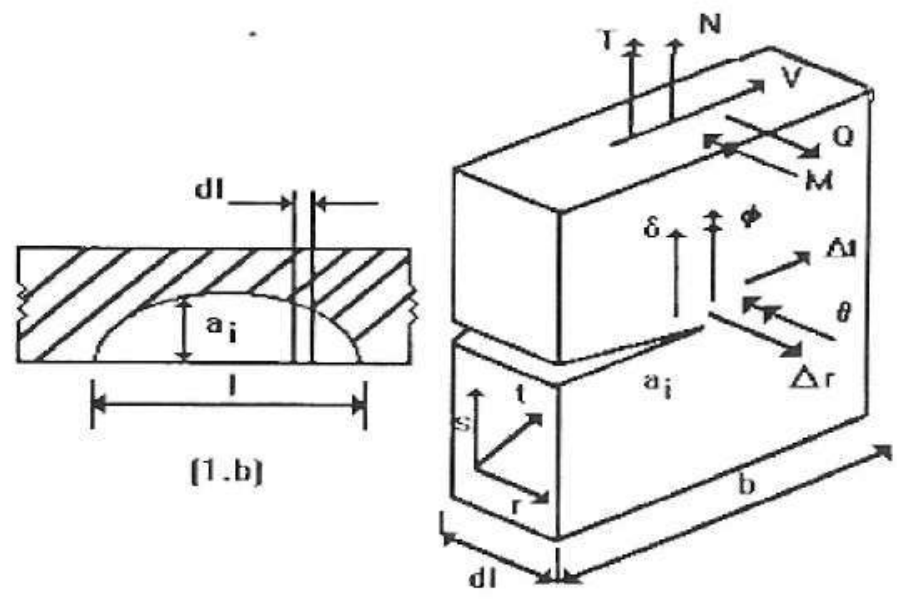

[1.c]

Figura 1 - llustraçăo do Modelo de Linha de Molas.

A idéia principal consiste em se considerar a trinca como passante, isto é, atravessando toda a espessura da casca. Os efeitos dos ligamentos da seção remanescente são então representados por uma série de molas que poderão absolver e transmitir esforços.

A Figura 1a mostra uma placa sujeita a cargas remotas em qualquer direção e em uma seção uma trinca não passante (Fig.1b). A placa é então discretizada por elementos finitos de casca e a seção remanescente é representada por uma linha de molas contínua, cuja de rigidez dependerá da profundidade da trinca em cada ponto, que é suposta igual de uma chapa de largura dl (Fig. 1c). Sabendo-se que existe uma relação entre os esforços e os deslocamentos relativos da face trincada, escreve-se para cada parcela dl a seguinte relação constitutiva:

$\left[\begin{array}{l}\delta \\ \theta \\ \Delta t \\ \Delta r \\ \phi\end{array}\right]=\left[\begin{array}{lllll}c_{11} & c_{12} & c_{13} & c_{14} & c_{15} \\ c_{21} & c_{22} & c_{23} & c_{24} & c_{25} \\ c_{31} & c_{32} & c_{33} & c_{34} & c_{35} \\ c_{41} & c_{42} & c_{43} & c_{44} & c_{45} \\ c_{51} & c_{52} & c_{53} & c_{54} & c_{55}\end{array}\right] \times\left[\begin{array}{c}N \\ M \\ V \\ Q \\ T\end{array}\right]$

As flexibilidades $c_{i j}$ de cada ponto ao longo da mola e suposta igual a de uma chapa com uma trinca simples, com profundidade $a_{i}$ e dimensões $b$ e dl como mostrada na Figura 1.c, cuja solução é conhecida e pode ser encontrada nos manuais de mecânica da fratura (Tada, 1983).
Percebe-se que os esforços de membrana (N) e de flexão (M) estão relacionados com os deslocamentos d e q e ao modo I de fratura; o esforço cisalhante (V) esta relacionado com o deslocamento $D_{t}$ e ao modo II; os esforços cisalhante $(\mathrm{Q})$ e de torgao $(\mathrm{T})$ estão relacionados com os deslocamentos $\mathrm{D}_{r}$ e q e ao modo III.

Por equilíbrio de forças na chapa da Figura $1 \mathrm{c}$, chega-se a:

$$
c_{13=} c_{14}=c_{15}=c_{23}=c_{24}=c_{25}=c_{34}=c_{35}=O
$$

Com isto a equação (1) pode ser invertida através de procedimentos matriciais elementares obtendo-se assim a matriz de rigidez da molas, que são adicionadas ao sistema de equações gerada pelo método dos elementos finitos, cuja solução é o campo de deslocamentos nodais.

Obtido os deslocamentos, o fator de intensidade de tensão para cada modo de fratura e dados por:

$$
\begin{gathered}
K_{t}=b^{1 / 2}\left(g_{1} \frac{N}{b}+g_{?} \frac{6 M}{b^{2}}\right) \\
K_{t}=b^{1 / 2}\left(g_{5} \frac{V}{b}\right) \\
K_{a t}=b^{1 / 2}\left(g_{4} \frac{Q}{b}+g_{5} \frac{6 T}{b^{2}}\right)
\end{gathered}
$$

Onde g. sao funções apenas da geometria da trinca e são encontradas em Tada (1983) e Desvaux (1985).

A taxa de energia dissipada na fratura é dada por 


$$
\mathscr{E}=\frac{\partial \Omega}{\partial a}=\frac{K_{1}^{2}+K_{1}^{2}+}{E^{\prime}}+\frac{K_{m}^{2}}{2 G^{\prime}}
$$

sendo $E^{\prime}=E /\left(I-v^{2}\right) ; G^{\prime}=E / 2\left(I+v^{\prime}\right)$ e $E$ o modulo de elasticidade longitudinal e $n$ o coeficiente de Poisson. Substituindo-se as equações (3), (4) e (5) em (6) e aplicando-se o Teorema de Castigliano, chega-se as flexibilidades da linha de molas

$$
c_{i j}=\frac{2}{E^{\prime}} \int_{j}^{u} g_{v} g_{k t} \partial u
$$

\section{PROCEDIMENTO PARA FADIGA}

Uma das vantagens em se utilizar o MLM para a propagação de trincas por fadiga e que após a discretização inicial do problema e de uma provável região de crescimento lateral da trinca como mostrado na Figura 2. Toda a análise é processada sem necessidade de modificações na malha ou a interferência do analista. A profundidade da trinca na região de predição de crescimento lateral deve ser da ordem de 1/1000 da espessura da casca, e faz-se necessário uma verificação inicial para que os valores de DK nesta região deverá ser menor que $\mathrm{D} K_{f}$ para que se tenha uma boa aproximação do comportamento real da trinca. Com isto, temse a possibilidade da trinca crescer tanto na direção da profundidade quanto na direção lateral.
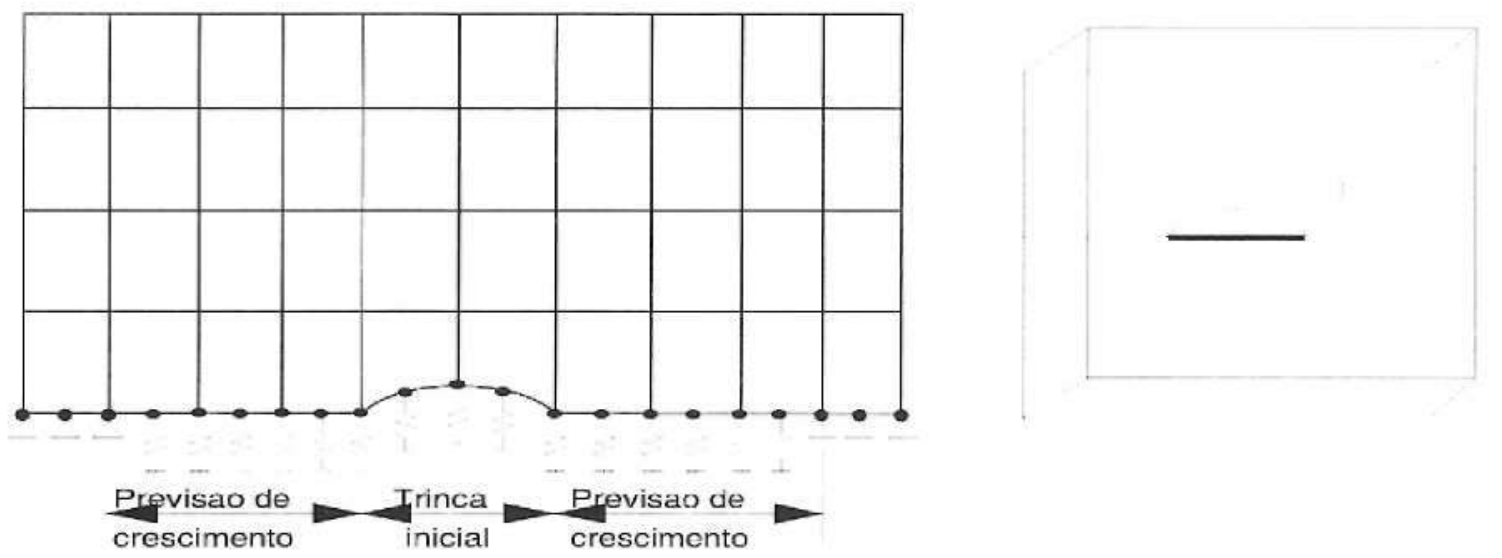

Figura 2 - llustração do MLM para fadiga.

A determinagao do numero de ciclos que a estrutura ira suportar (vida util), sera feita por integragao da iei de Paris (1965), que apresenta bons resultados e muito utilizada para este tipo de problema.

$$
d a / d N=C(\Delta K)^{n}
$$

onde a e a profundidade da trinca; No numero de ciclos; $C$ e n sao constantes do material e DK e a variagao do fator de intensidade de tensao em um ciclo de carregamento dado por

$$
\Delta K=K_{\max }-K_{m i n}
$$

A relagao (8) e escrita de forma incremental e aplicada a cada mola. Adotando-se um incremental de ciclo DN, assim obtém-se,

$$
\Delta a=\Delta N C(\Delta K)^{n}
$$

e apos a k-esima iteração a profundidade da trinca em cada ponto ao longo da linha de molas e atualizada por:

$$
a^{k+1}=a^{k}+\Delta a^{k}
$$

com a profundidade da trinca atualizada, nova análise é processada até que uma condição crítica pré determinada pelo analista seja alcançada, que podem ser:

Uma profundidade crítica ou máxima $a_{i}$.

Um determinado numero $N$ de ciclos.

Um valor de $K_{\max }$ maior do que a tenacidade do material.

A numeração dos nós da trinca deve preferencialmente ser deixada por último, isto otimiza o processarnento dos dados e obtém-se maior desempenho do solve-parcial.

\section{ANÁlise EM REGIME ELASTO-PLÁSTICO}

O sistema permite uma análise completa de toda a estrutura em regime elasto-plástico, entretanto para estruturas com trincas localizadas, devido a alta concentração de tensão gerada na região ao redor da trinca, pode-se realizar uma 
análise com bom grau de precisao considerandose apenas a não linearidade física somente nesta região.

Em regime elasto-plástico a equação (6), que tem o mesmo valor do que o integral-J, definida por Rice, e escrita na forma incremental e as características elásticas dependerão da história do carregamento. Algumas constantes relativas ao material deverão ser adequadamente ajustadas (Parks, 1981; Parks \& White, 1983). Como parâmetro de fratura é avaliado o integral-J, que será feito em duas etapas distintas, dividindo-se em uma parcela elástica Je e a outra plástica Jp, como:

$$
\mathbf{J}=\mathbf{J e}+\mathbf{J p}
$$

A parcela Je e proporcional ao quadrado do FITs e sera dado pela relação (6) e a parcela Jp é determinada incrementalmente, podendo ser expressa de forma expedita em função do deslocamento plástico na extremidade da trinca (Sp), assim escreve-se:

$$
\mathbf{J} \mathbf{p}=m \sigma_{p} \delta_{i}
$$

sendo $s_{\mathrm{p}}$ a tensão de escoamento do material e $m$ uma constante de forma.

\section{CARACTERISTICAS DO SISTEMA SHELLFRAT}

O sistema SHELLFRAT foi escrito em linguagem FORTRAN e pode ser processado tanto em sistema de grande porte quanto em micro computadores tipo PC.

Trata-se de um programa de elementos finitos sendo que para a discretização da superfície são utilizados elementos quadráticos tridimensional degenerados para a superfície média da casca, que permite representar superfícies com qualquer curvatura e espessuras variáveis, apresentando uma rápida convergência tanto para cascas finas como espessas.

Suas principais características são:

- Elemento tridimensional de casca

- Elemento de linha de molas para trincas

- Alocação dinâmica de memória.

- Geração semi-automática de coordenadas, incidências e condições de contorno.

- Armazenamento da banda superior da matriz de rigidez por blocos de altura efetiva.

- Solução do sistema de equações por blocos.

- Solve-parcial

- Método de Newton Raphson para a solução não linear.
- Criterio plástico de Von-Mises.

- Interface gráfica com GID.

\section{APLICAÇÕES NUMÉRICAS}

\subsection{Chapa com Trinca Semi Elíptica}

A primeira aplicação corresponde ao clássico problema de uma chapa contendo uma trinca não passante, de formato semi-elíptico, considerada como trinca curta, submetida a cargas de tração e flexão, semelhante a mostrada na figura 1 a. Adotou-se as seguintes dimensões (em $\mathrm{mm}$ ) e características físicas: $\mathrm{a}=14 ; 2 \mathrm{c}=70 ; \mathrm{b}=28 ; \mathrm{W}=$ 840 (largura da chapa); $L=1148$ (comprimento chapa); $\mathrm{E}=2,06 \times 10^{5} \mathrm{~N} / \mathrm{mm}^{2} ; v=0,3 ; \Delta K_{1 \mathrm{I}}=60 \mathrm{~N} / \mathrm{mm}^{3 / 2}$; $\mathrm{K}_{\mathrm{lc}}=5000 \mathrm{~N} / \mathrm{mm}^{3 / 2} ; C=6,94 \times 10^{-1.3}$ e $n=2,67$. Na modelagem do problema, levou-se em conta a dupla simetria, assim utilizou-se de 59 elementos de casca e 10 elementos de linha de molas ( 5 para a trinca e 5 para previsão do crescimento lateral da trinca). A malha está mostrada na Figura 3. A chapa foi submetida a três tipos de carregamentos: tração simples com $\sigma_{\mathrm{t}}=157 \pm 108 \mathrm{~N} / \mathrm{mm}^{2}$; flexão pura com $\sigma_{1}=157 \pm 108 \mathrm{~N} / \mathrm{mm}^{2}$ e flexo-traçăo com $\sigma_{\mathrm{ft}}=$ $108108 \mathrm{~N} / \mathrm{mm}^{2}$.

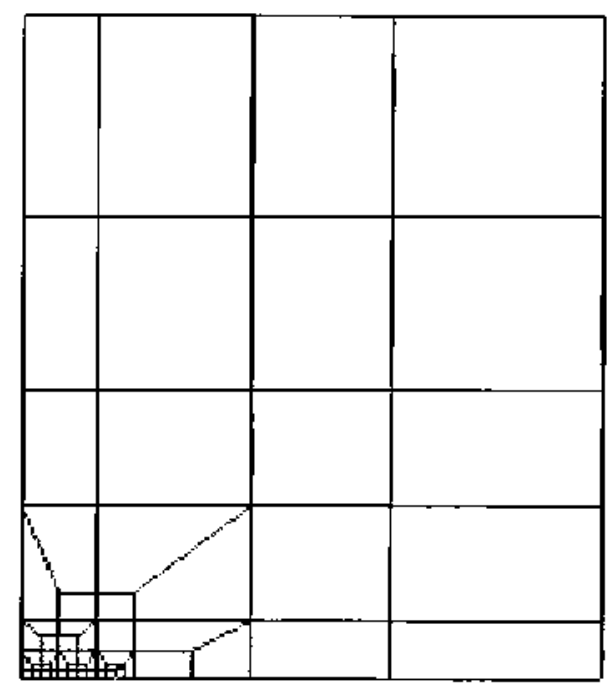

Figura 3 - Malha da chapa analisada.

Os resultados para o ponto de máxima profundidade e comprimento da trinca são mostrados nas Figuras 4 e 5 respectivamente. $E$ apresentaram uma boa concordância com os obtidos por Aamodt (1974), utilizando uma formulação tridimensional do MEF. Percebe-se que para trincas curtas sob tração a trinca se propaga com maior velocidade na direção da profundidade e sob flexão a propagação se da com mais intensidade ao longo do comprimento da trinca. 


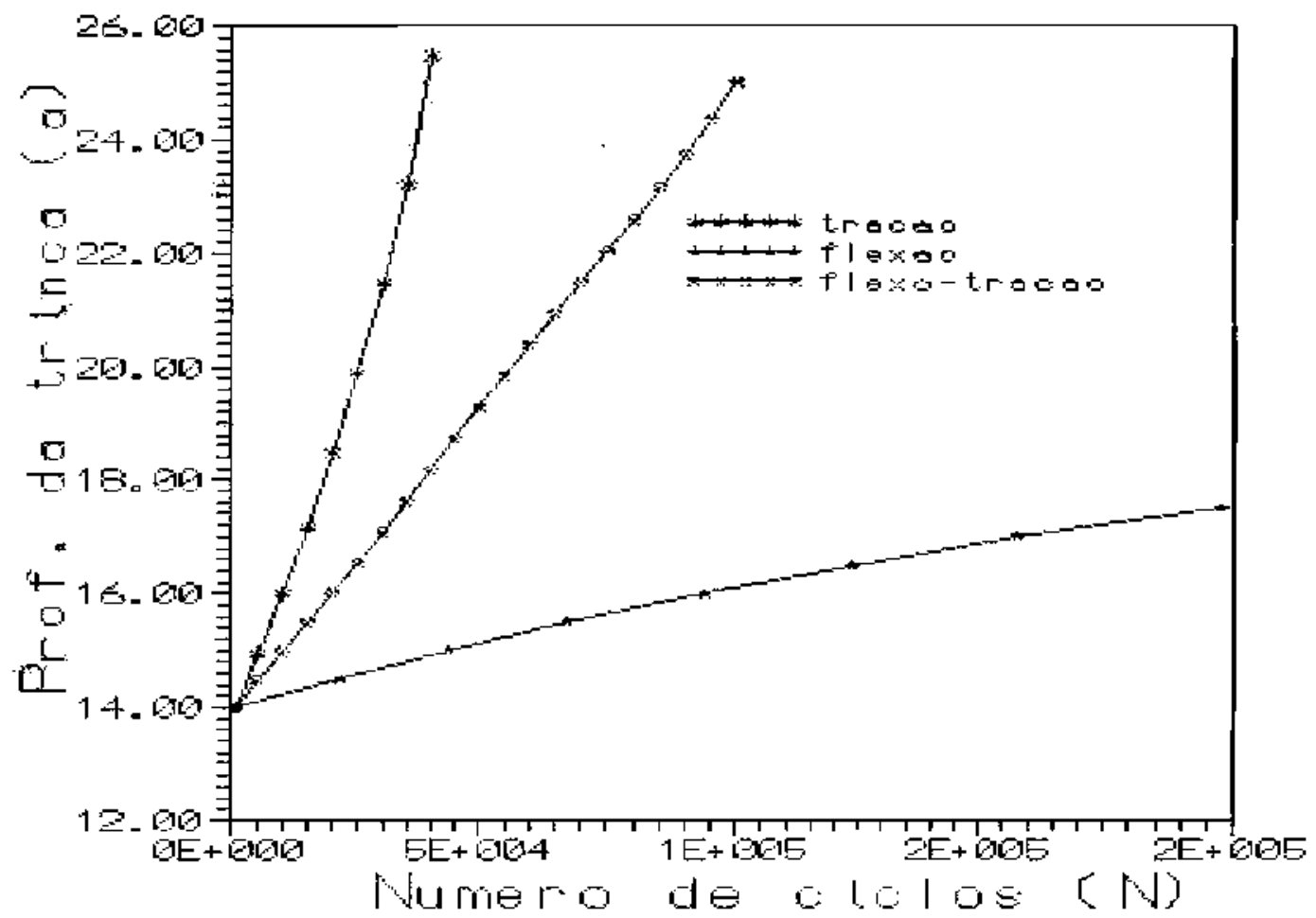

Figura 4 - Profundidade máxima $\alpha \times$ Número de Ciclos.

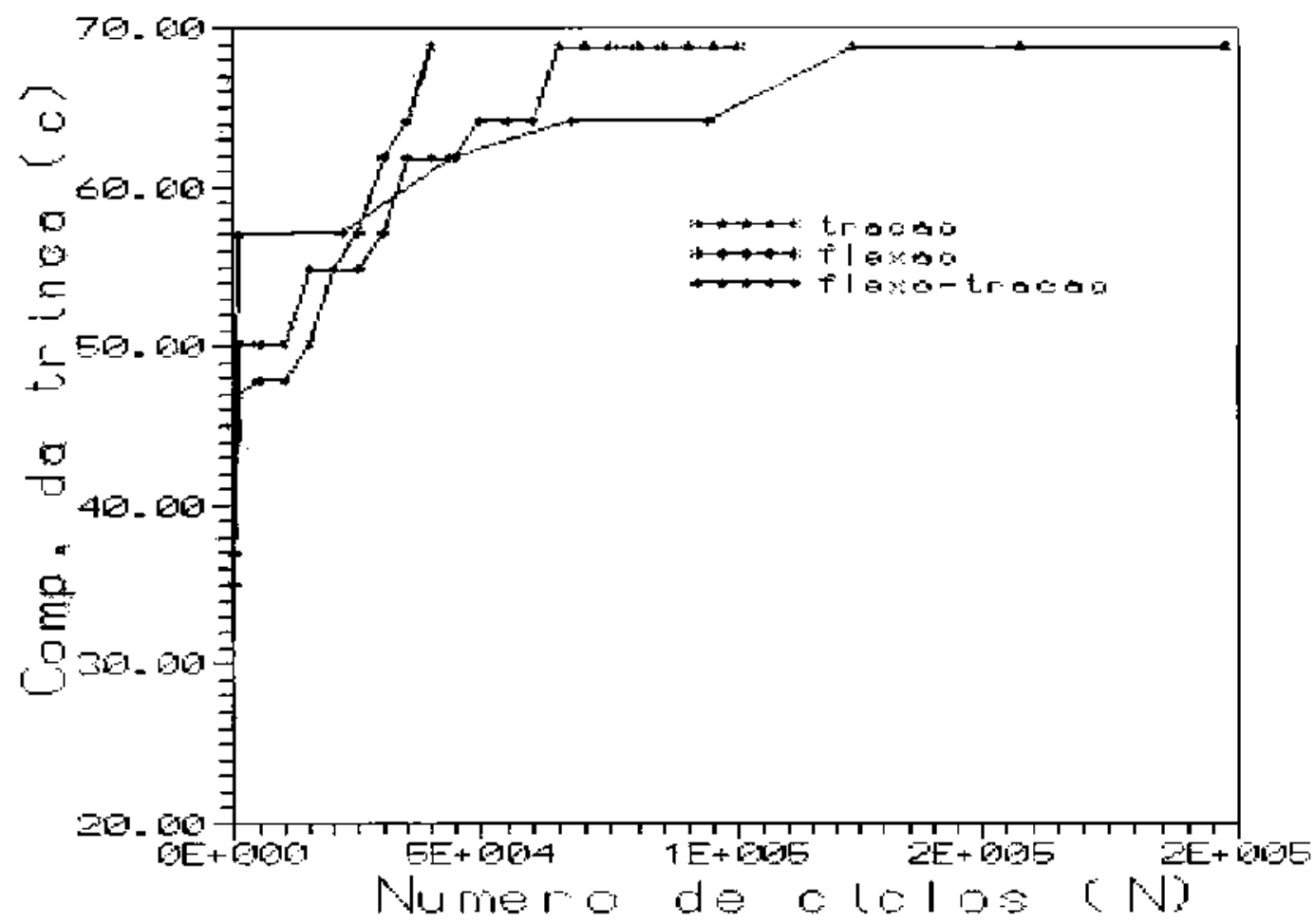

Figura 5 - Semi comprimento $c$ x Número de Ciclos. 


\subsection{Junta T com trinca na junção dos tubos.}

Analisa-se a vida útil a fadiga de uma típica junta tubular de plataforma marítima de extração de petróleo. A trinca esta localizada na junção do tubo principal com o secundário. As características do materia! são; $\mathrm{E}=2,10 \times 10^{5} \mathrm{~N} / \mathrm{mm}^{2} ; \mathrm{n}=0,3$;
$A K_{l f}=100 \mathrm{~N} / \mathrm{mm}^{3 / 2} ; \mathrm{K}_{1 \mathrm{c}}=5000 \mathrm{~N} / \mathrm{mm}^{3 / 2} ; C=3, \mathrm{Ox} 10^{-13}$ e $n=3,00$. A carga estática aplicada foi de $500 \mathrm{kN}$ distribuído no perímetro do tubo secundário e para a analise da vida útil aplicou-se $D F=250 \pm 250 \mathrm{~K} \mathrm{~N}$. A geometria é mostrada na Figura 6 e malha usada na modelagem é mostrada na Figura 7.

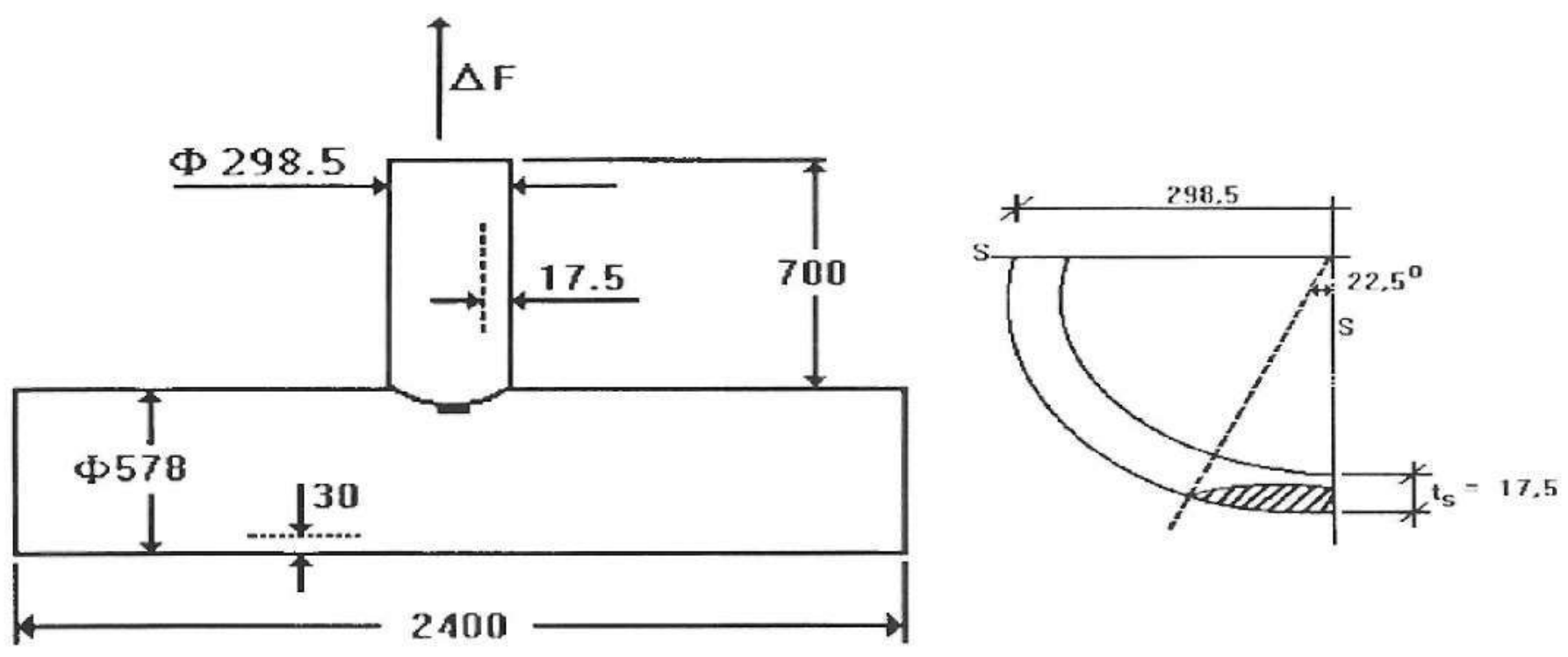

Figura 6 - Geometria da junta T e detalhe da trinca.

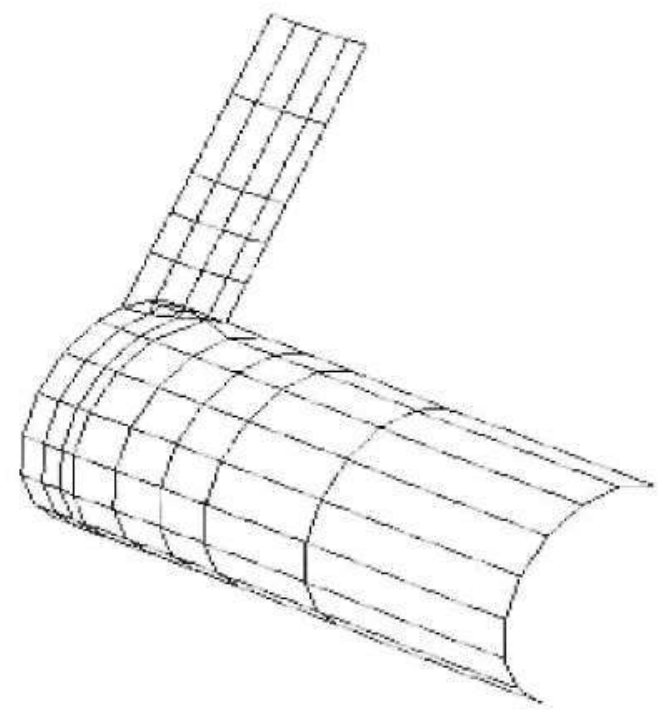

Figura / - Malna utılızada na analıse da junta $T$.

A Figura 8a), mostra a variação do fator de intensidade de tensão ao longo da trinca na configuração inicial, percebe-se pelos resultados a predominância do modo 1 de fratura. Na Figura 8b) está mostrado a propagação de trinca na direção da profundidade máxima. A trinca tornouse passante após aproximadamente 200000 ciclos de carregamento. Estes resultados concordam com avaliações realizadas com auxilio da norma PD6493 (1980). 


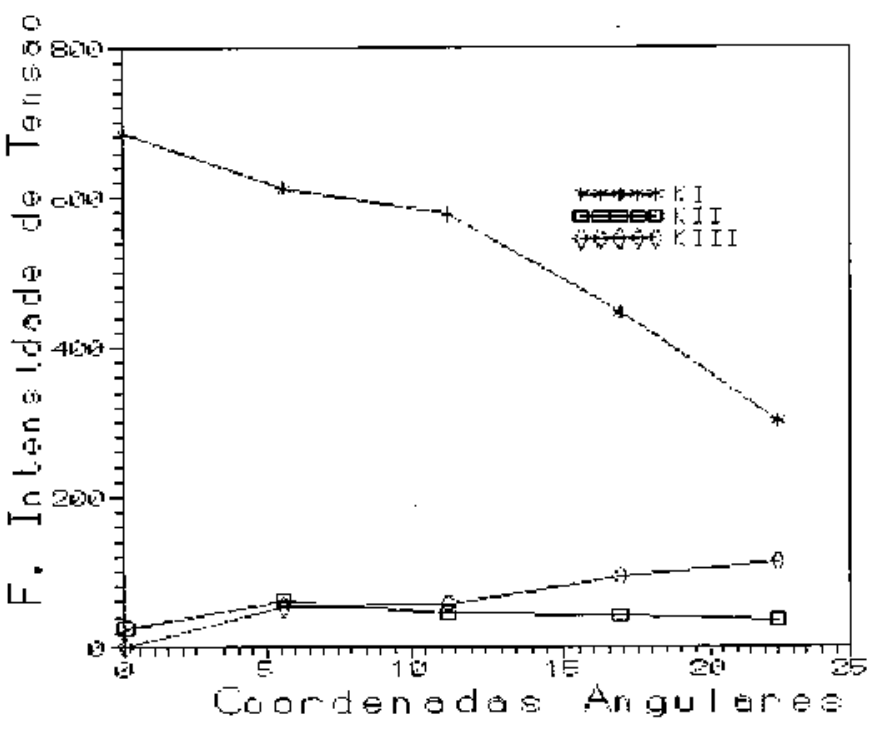

a) Fator de Intensidade de Tensão.

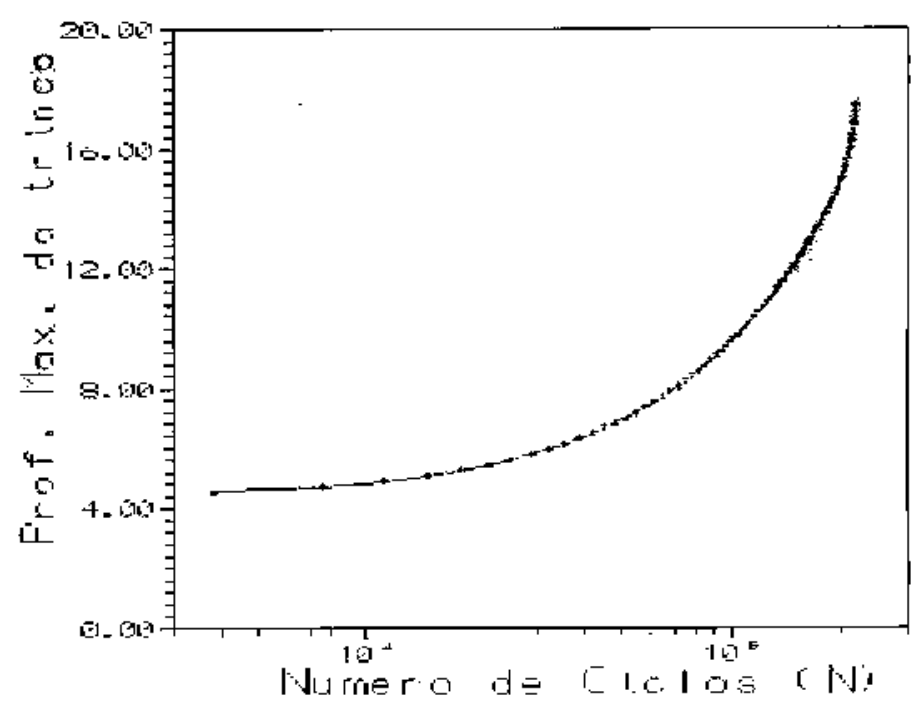

b) Profundidade da trinca $\times$ Ciclos de carga.

Figura 8 - Resultados para a Junta T.

\section{CONCLUSÕES}

Mostrou-se aqui que é possível a análise de um problema bastante complexo de estruturas em casca com trincas não passantes, com auxílio do sistema SHELLFRAT. Os exemplos numéricos mostraram a análise para comparação de um problema clássico e uma típica aplicação de engenharia, permitindo avaliar a estrutura pós trincamento. Podese assim perceber as potencialidades do MLM e do sistema SHELLFRAT. Análise de situações reais com maior grau de complexidade, tais como modos mistos de fratura, carregamentos aleatórios, trincas em formatos não elíptico e outros; podem ser tratados sem maiores dificuldades. A utilização do MEF junto com o MLM possibiiita a análise de forma simples e segura de estruturas contendo trincas não passantes. Na avaliação da vida útil de estruturas obtem-se soluções para muitos casos práticos com simplicidade, economia e bom grau de precisão. Tornando-se assim uma ferramenta versátil, segura e de facil utilização, obtendo-se soluções para muitos casos práticos com simplicidade, economia e bom grau de precisão.

SOUZA, L. A. de; EBECKEN, N. F. F. SHELLFRAT A computational system/code for analysis of PartCracked Surface Shell Structures. Semina: Ci. Exatas/Tecnol. Londrina, v. 21, n. 4, p. 9-16, dez. 2000.

ABSTRACT: This work presents the SHELLFRAT computational system, based on the Finite Element Method for analysis of part-cracked surface shell structure. The structure is modeled by shell elements and the crack is simulated by spring elements which represent cracks with variable shapes, directions and depths. The system can process elastic or elastic-plastic analyses,considerations in mixed mode fracture, simulate the propagation of the cracks due to constant use and anticipate the lifetime of the structure. As a result of the analysis, the tension intensity factors are evaluated, as well as the integral J, the COD-crack opening displacement, final crack geometry and number of cycles of load.

KEY WORDS: finite element, shell structures, cracks, stress intensity factor, constant use. 


\section{REFERÊNCIAS BIBLIOGRÁFICAS}

AAMODT, B. Application of the Finite Element Method to Problems in Linear and Non Linear Fracture Mechanics. Norway: Ed. University of Trondheim, 1974.

DELALE, R; ERDOGAN, F. Line-Spring Model for Surface Crack in a Reissner Plate. Int. J.Engng. Sci, v. 19, p.13311340,1981 .

DESVAUX, J. D. The Line Spring Model For Surface Flaw, An Extension To Mode II And Mode III", M.Sc. Thesis, M.I.T., 1985.

GERMAN, M. D.; KUMAR, V.; LORENZI, H.G. Analysis of Surface Cracks in Plates and Shells Using the Line - Spring Model and Adina. Computers \& Structures, v. 17, p. 881-898, 1982.

IRWIN, G. R. Analysis of Stresses and Strains Near the End of a Crack Transversing a Plate. Trans, of A.S.M.E., J. Appl. Mech., 1957.

OLOWOKERE D. O.; NWOSU, D.I. Numerical Studies on Crack Growth in a Steel Tubular T-Joint. Int. J. Mech. Sci, n.7, p 859-871, 1997.

PARIS, P. C; ERDOGAN F. A Critical Analysis of Crack Propagation Laws. T. of ASME, J. Bas. Eng., p. 528, 1963.

PARKS, DM. The Inelastic Line-Spring: Estimates of ElasticPlastic Fracture Mechanics Parameters for Surface-Cracked Plates and Shells. Trans, of ASME J.P.V.T., v. 103, p. 246 254,1981 .
PARKS, D.M.; WHITE, C.S. Elastic-Plastic Line-Spring Finite Elements for Surface Cracked Plates and Shells Plates and Shells. Trans, of ASME J.P.V.T., v. 104, p. 286-292,1982

PD6493 - Guidance on some Methods for the Derivation of Acceptance Levels for Defects in Fusion Welded Joints. [S. I.]: British Standards Institution, 1980.

RICE, J. R. A Part Independent Integral and the Aproximate Analysis of Strain Concentration by Notches and Cracks. Trans. of ASME, J. Appl. Mech., v. 35, 1968.

RICE, J. R.; LEVY, N. The Part-through Surface Crack in a Elastic Plate, T of A.S.M.E., J. Appl. Mech, v. 19, 185-194, 1972.

SKALLURED, B. Numerical Analysis of Cracked Inelastic Shells with Large Displacements or Mixed Mode Loading. Int. J. Solid and Structures, v. 36, p. 2259-2283, 1999.

SOUZA, L. A.; EBECKEN, N. F. F. A Finite Element Program of Crack-Growth in Shell Structures. In: COMPUTATIONAL Plasticity, Models, Software and Application. Swansea: Pineridge Press, 1987.

SOUZA L. A. The line Spring Model for Part-Through Cracks Analysis in Mixed Modes with Consideration of Plasticity and Fatigue. 1993. D.Sc. Thesis - COPPE/UFRJ, Brasil, 1993. (in Portuguese)

TADA, M., PARIS, P.; IRWIN, G. The Stress Analysis of Cracks Handbook. [S.I.]: Del Research Corporation, 1983. 